\title{
Redox-Active Phenanthrenequinone Triangles in Aqueous Rechargeable Zinc Batteries
}

Kwan Woo Nam,${ }^{\dagger}$ Heejin Kim,${ }^{\perp}$ Yassine Beldjoudi, ${ }^{\dagger}$ Tae-woo Kwon, ${ }^{\dagger}$ Dong Jun $\mathrm{Kim}^{\# *}$ and J. Fraser Stoddart ${ }^{\dagger, \#, \S *}$

$\dagger$ Department of Chemistry, Northwestern University, Evanston, IL 60208, USA

${ }^{\perp}$ Division of Analytical Science, Korea Basic Science Institute, 169-148 Gwahak-ro, Yuseong-gu, Daejeon 34133, Republic of Korea

\#School of Chemistry, UNSW Sydney NSW 2052, Australia

§Institute for Molecular Design and Synthesis, Tianjin University, 92 Weijin Road, Nankai District, Tianjin 300072, China

*E-mail: dongjun.kim@unsw.edu.au; stoddart@northwestern.edu

\section{Supplementary Information}

\section{Table of Contents}

$\begin{array}{ll}\text { Section A. Materials and Characterization } & \text { S2 }\end{array}$

$\begin{array}{ll}\text { Section B. Physical Characterization } & \text { S3 }\end{array}$

$\begin{array}{ll}\text { Section C. Electrochemical Measurements } & \text { S5 }\end{array}$

$\begin{array}{ll}\text { Section D. References } & \text { S7 }\end{array}$ 


\section{Section A. Materials and Characterization}

\section{1) Materials}

All commercially available reagents and solvents were purchased from Sigma Aldrich and used as received without further purification. Zn film, Steel Use Stainless (SUS) film, and coin cells obtained from Goodfellow and Pred Materials, respectively. PQ- $\Delta$ was prepared according to the previous reported procedure, ${ }^{1}$ washed with water and acetone respectively, and dried in air.

\section{2) Characterization}

For the characterization of PQ- $\Delta$ at different charge and discharge states, the cells were opened and rinsed with DI water inside a glove box. In order to obtain the water content, thermogravimetric analysis (TGA, Netzsch) was carried out from room temperature to $300{ }^{\circ} \mathrm{C}$ at a ramping rate of $5{ }^{\circ} \mathrm{C} \mathrm{min}^{-1}$ under an Ar flow.

Powder X-ray diffraction (PXRD, STOE STADI-P) with $\mathrm{Cu}-\mathrm{K} \alpha 1$ radiation $(\lambda=1.54056 \AA$ ) was measured through transmission geometry for crystal structure analysis by scanning in the $2 \theta$ range of $10^{\circ}-50^{\circ}$ with accelerating voltage and current of $40 \mathrm{kV}$ and $40 \mathrm{~mA}$. For the ex situ PXRD characterization of PQ- $\Delta$ at different charge and discharge states, the cells were opened and rinsed with DI water inside a glove box.

The mass of PQ- $\boldsymbol{\Delta}$ was confirmed by matrix-assisted laser desorption/ionization-time of flight (MALDI-TOF, Bruker AutoFlex-III) using laser of $355 \mathrm{~nm}$ and $\alpha$-cyano-4-hydroxycinnamic acid (CHCA) as a matrix.

The oxidation states of electrodes were analyzed by X-ray photoelectron spectroscopy (XPS, Thermo Scientific ESCALAB 250Xi). Each sample was dried under vacuum for $1 \mathrm{~h}$ prior to XPS measurements. For the ex situ XPS characterization of PQ- $\Delta$ at different charge and discharge states, the cells were opened and rinsed with DI water inside a glove box.

UV-Vis-NIR Spectra were recorded on a Shimadzu UV-3600 spectrophotometer. 


\section{Section B. Physical Characterization}

\section{1) MALDI-TOF analysis of $P Q-\Delta$}

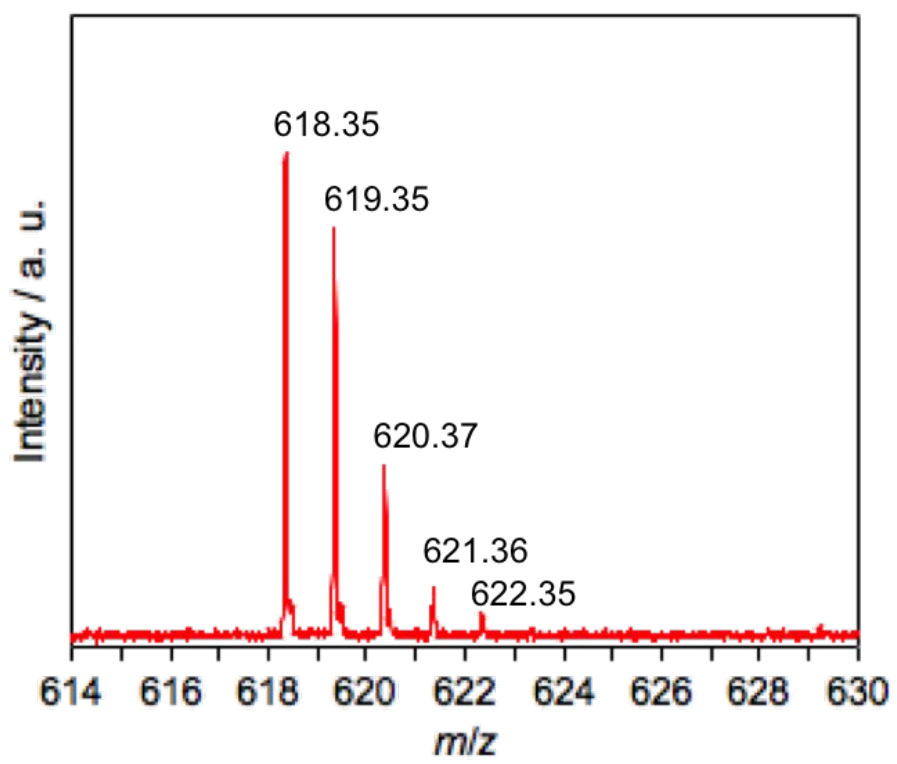

Figure S1. MALDI-TOF Mass spectra of PQ- $\Delta$, measured with $\alpha$-cyano-4-hydroxycinnamic acid (CHCA) as a matrix.

\section{2) Ex situ PXRD analysis of $P Q-\Delta$ electrode}

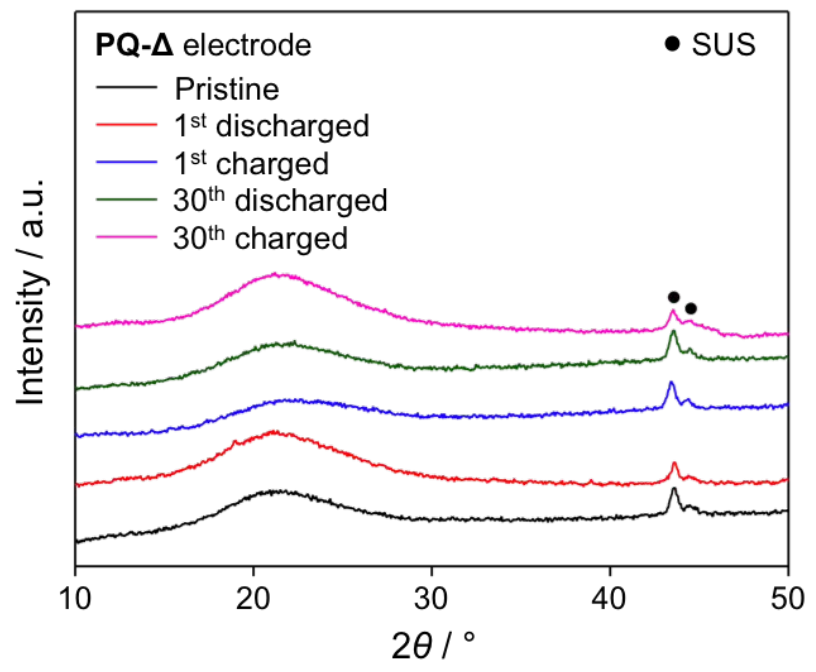

Figure S2. PXRD patterns of the PQ- $\Delta$ electrode in the pristine, $1^{\text {st }}$ and $30^{\text {th }}$ fully discharged/charged states at a rate of $30 \mathrm{~mA} \mathrm{~g}^{-1}$, respectively. 


\section{3) Ex situ XPS analysis of PQ- $\Delta$ electrode}
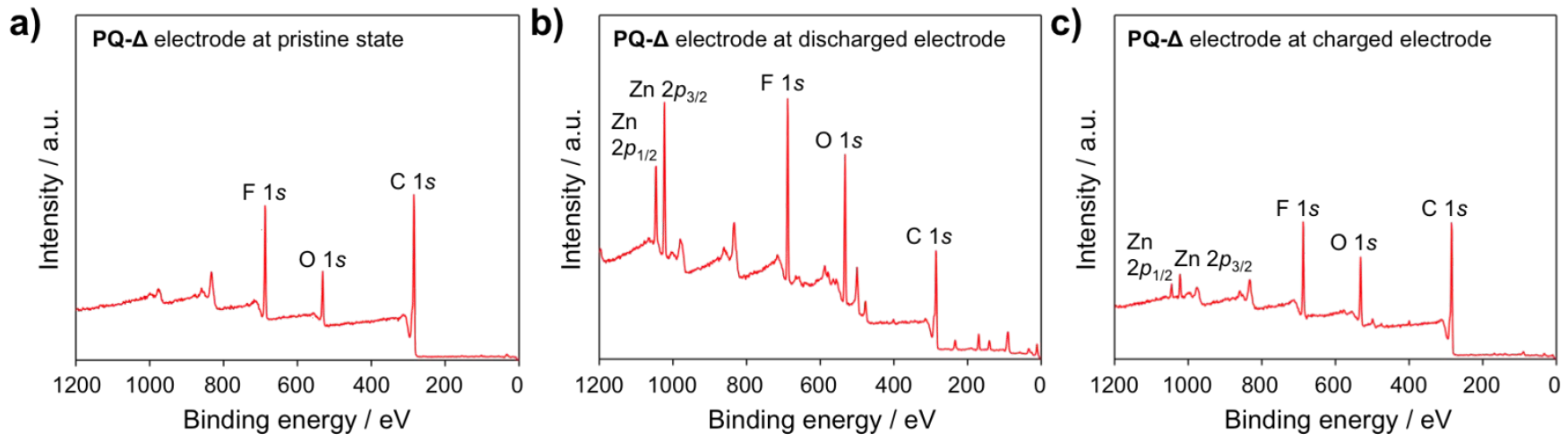

Figure S3. Ex situ XPS survey spectra of PQ- $\boldsymbol{\Delta}$ at (a) pristine, (b) discharged, and (c) charged electrodes.

\section{4) Ex situ UV-Vis analysis of $P Q-M$ and $P Q-\Delta$ electrodes}
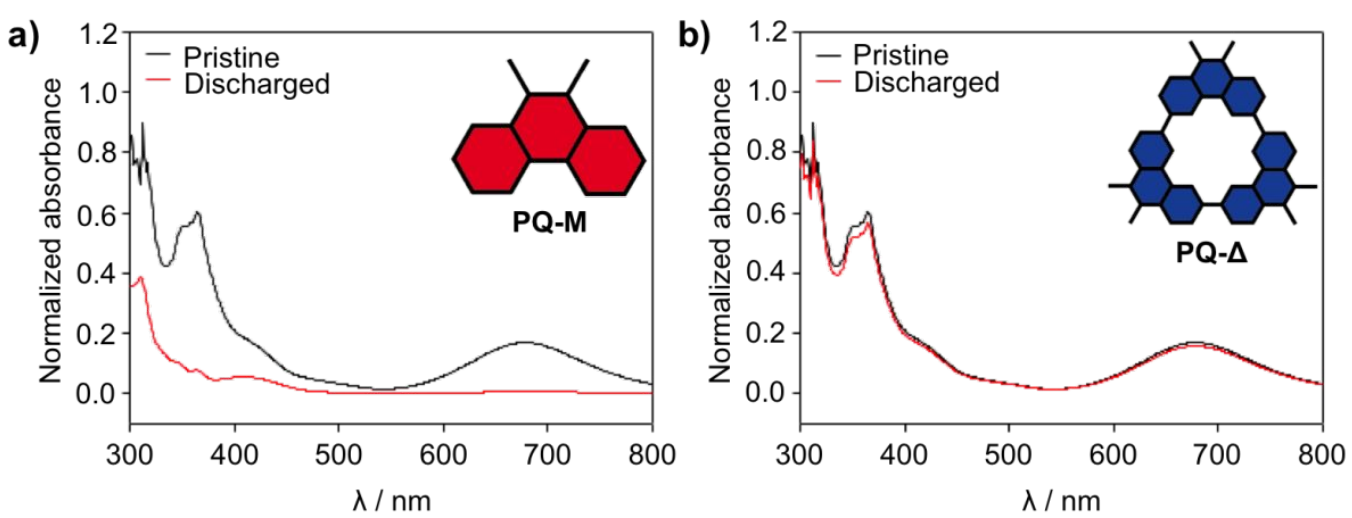

c)

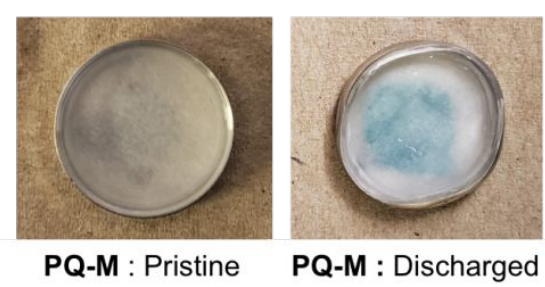

d)

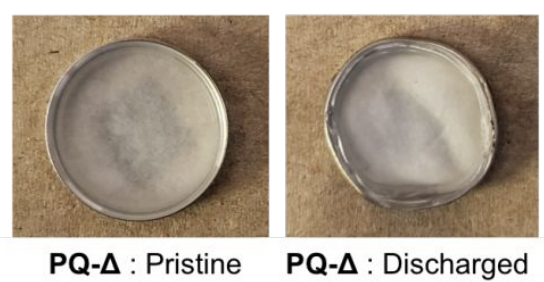

Figure S4. UV-Vis spectrophotometry performed on the pristine and the first discharged of (a) PQ-M and (b) PQ- $\Delta$. Digital photos of coin cells for the pristine and the first discharged of (c) PQ-M and (d) PQ- $\Delta$. Electrolyte: $3 \mathrm{M} \mathrm{Zn}\left(\mathrm{CF}_{3} \mathrm{SO}_{3}\right)_{2}$ in DI water. 
5) Ex situ FT-IR analysis for the separators and Zn metal of PQ-M

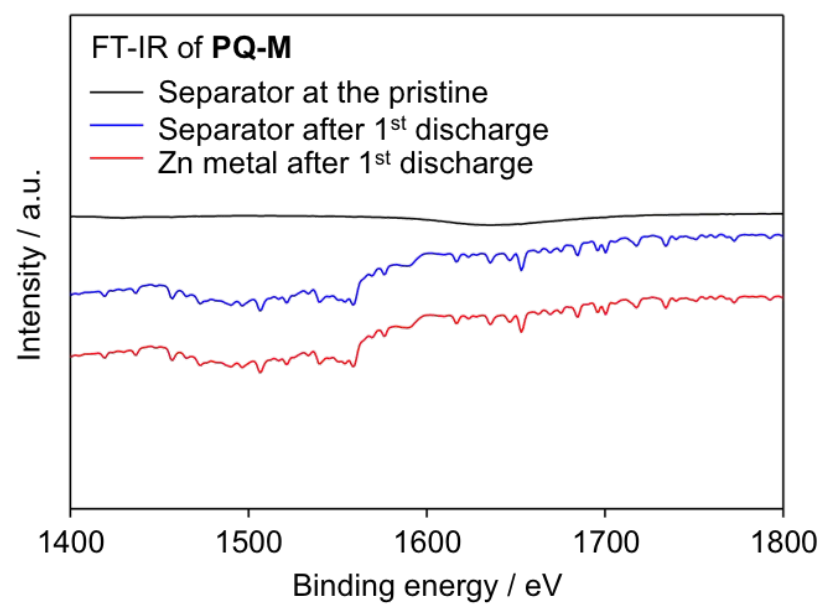

Figure S5. Ex situ FT-IR for the separators and Zn metal of PQ-M at the various states.

\section{Section C. Electrochemical Measurements}

1) Cyclic voltammogram of $P Q-\Delta$
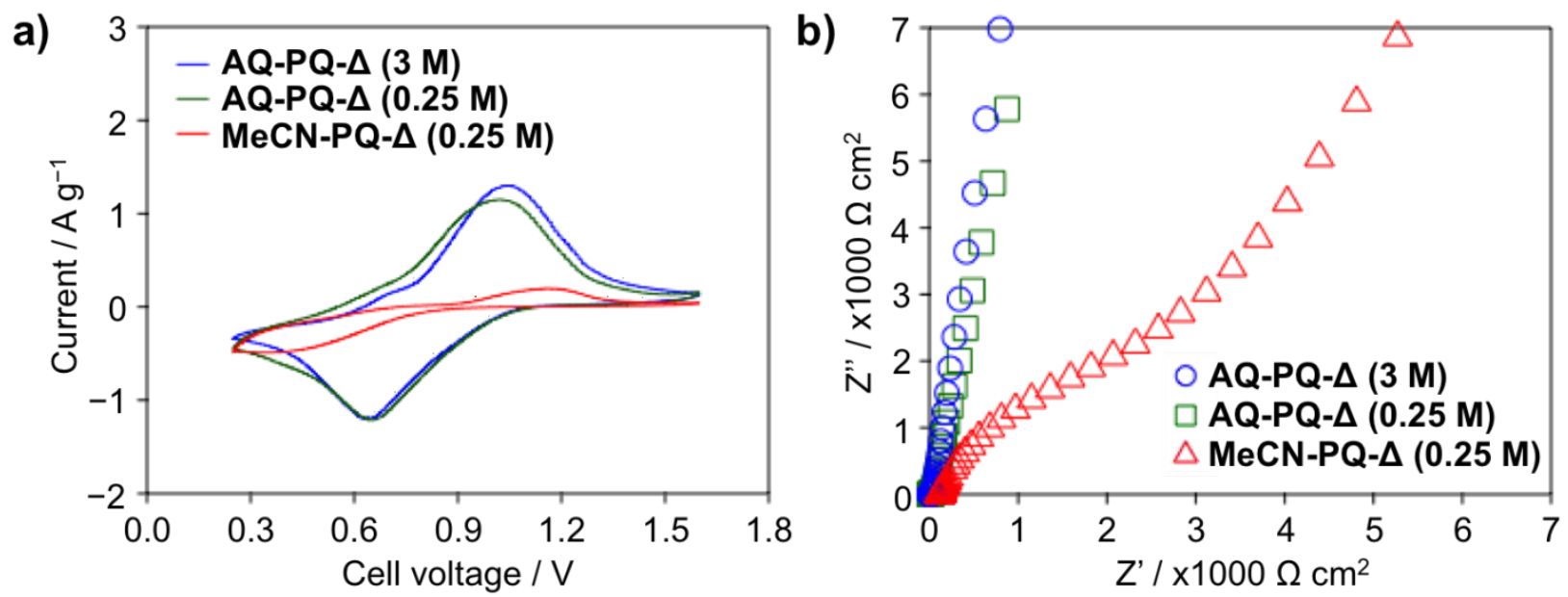

Figure S6. CV and EIS spectra profiles of PQ- $\boldsymbol{\Delta}$ using low (0.25 M) and high (3 M) electrolyte concentrations. Electrolyte: $0.25 \mathrm{M} \mathrm{Zn}\left(\mathrm{CF}_{3} \mathrm{SO}_{3}\right)_{2}$ in $\mathrm{MeCN}$ or $0.25 / 3 \mathrm{M} \mathrm{Zn}\left(\mathrm{CF}_{3} \mathrm{SO}_{3}\right)_{2}$ in $\mathrm{DI}$ $\mathrm{H}_{2} \mathrm{O}$. CV and EIS measurements were performed using a coin-type cell, two electrode configuration and symmetric cells, respectively, with the active electrode composed of PQ- $\mathbf{\Delta}$ : acetylene black : PVDF $=6: 3: 1$. 


\section{2) Electrochemical performance of $A Q-P Q-\Delta$}
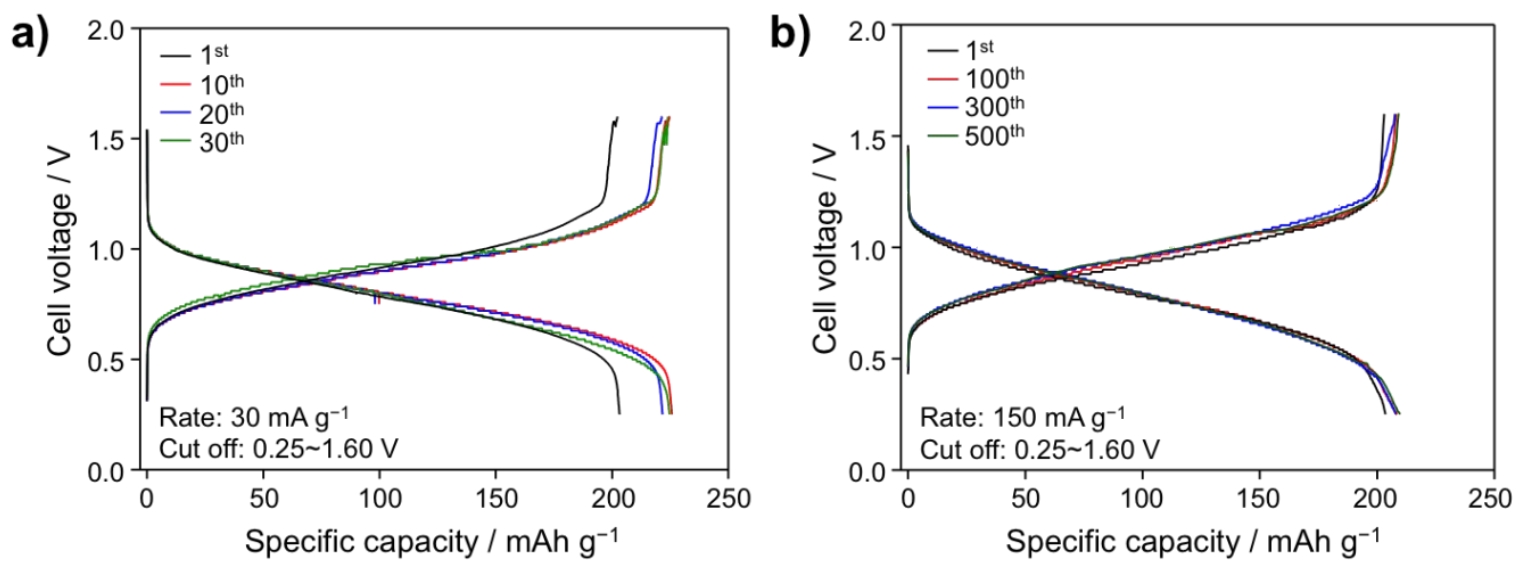

Figure S7. Discharge-charge of AQ-PQ- $\Delta$ with using aqueous electrolytes at a current density of (a) $30 \mathrm{~mA} \mathrm{~g}^{-1}$ and (b) $150 \mathrm{~mA} \mathrm{~g}^{-1}$. The discharge-charge process was performed using a cointype cell, two-electrode configuration with the active electrode composed of PQ- $\mathbf{\Delta}$ : acetylene black : PVDF $=6: 3: 1$. Electrolyte: $3 \mathrm{M} \mathrm{Zn}\left(\mathrm{CF}_{3} \mathrm{SO}_{3}\right)_{2}$ in $\mathrm{DI}_{2} \mathrm{O}$.

\section{3) Comparison of electrochemical performance as a function of molecular structures}

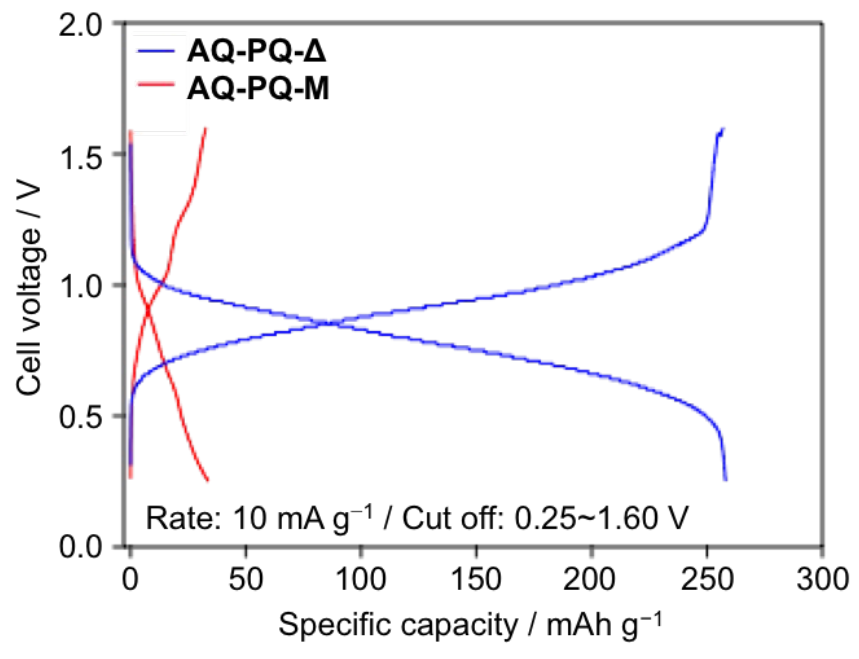

Figure S8. The voltage profiles of PQ-M and PQ- $\mathbf{\Delta}$ using aqueous electrolytes at a current density of $10 \mathrm{~mA} \mathrm{~g}^{-1}$ for both charge and discharge. The discharge-charge process was performed using a coin-type cell, two-electrode configuration with an active electrode composed of PQ-M or PQ- $\mathbf{A}$ : acetylene black : PVDF $=6: 3: 1$. Electrolyte: $3 \mathrm{M} \mathrm{Zn}\left(\mathrm{CF}_{3} \mathrm{SO}_{3}\right)_{2}$ in DI water. 
4) Ragone plots for the $\mathrm{Zn} / \mathrm{PQ}-\Delta$ battery

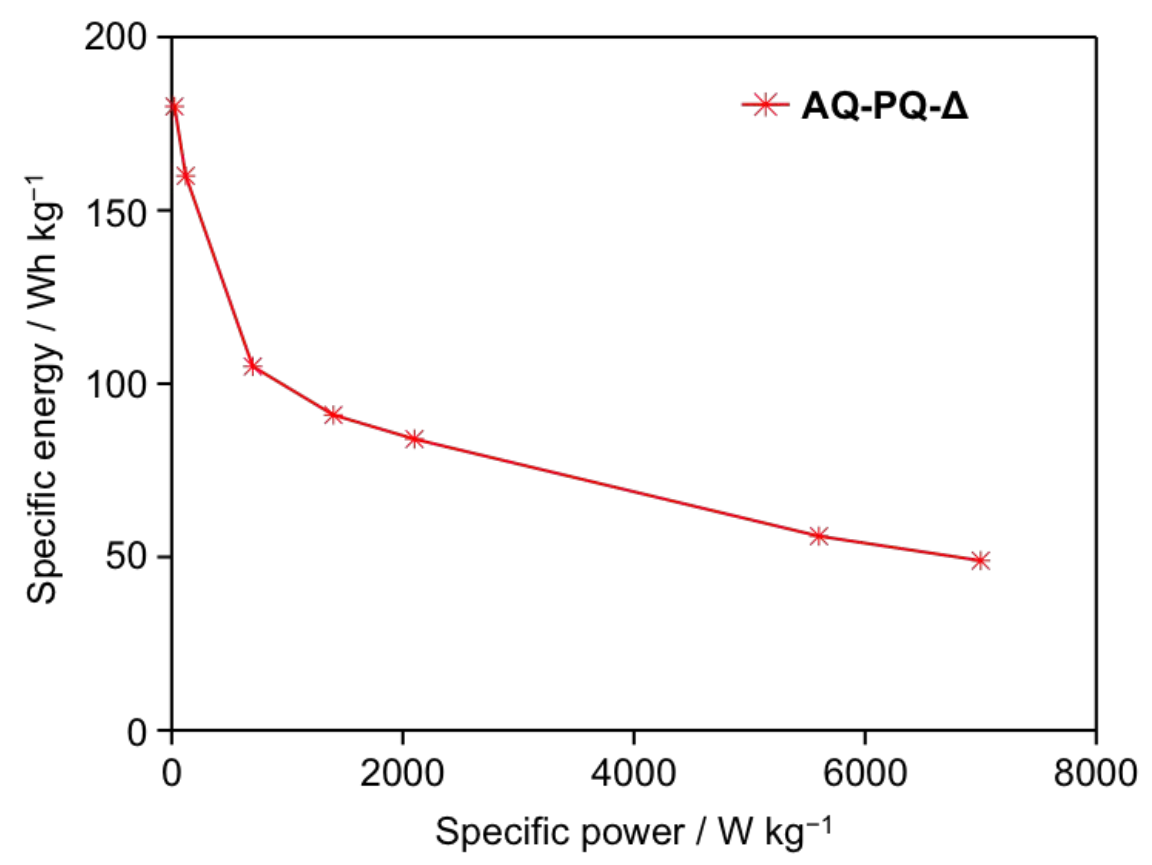

Figure S9. Ragone plots for the $\mathrm{Zn} / \mathbf{P Q}-\mathbf{\Delta}$ battery

\section{Section D. References}

(1) Zhang, J.; Wang, X.; Su, Q.; Zhi, L.; Thomas, A.; Feng, X.; Su, D. S.; Schlögl, R.; Müllen, K. Metal-Free Phenanthrenequinone Cyclotrimer as an Effective Heterogeneous Catalyst. J. Am. Chem. Soc. 2009, 131, 11296-11297. 